in the neonatal population. Preterm low birth weight neonates represent the group at highest risk of infection, mainly attributed to immunodeficiency present at birth. Antimicrobial therapy remains the main strategy for prophylaxis and treatment of infection. However, due to associated adverse effects, research into alternative therapeutic strategies such as immunoglobulin therapy has been prompted. One of the most important immune developments occurs intrauterine. Between weeks 30-32, transplacental transfer of maternal IgG to the foetus begins, conferring passive immunity. There is an incremental rise in foetal $\operatorname{IgG}$ with gestational age, thus preterm low birth weight neonates are born with a true deficiency of IgG antibodies. As low serum IgG has been reported to increase the risk of infection, IgG replacement therapy offers hope of enhancing immune competence and decreasing infectious episodes in this vulnerable population.

Aim We aimed to assess the efficacy of IVIG therapy in the prophylaxis of infection in preterm and low birth weight infants.

Methods All published studies of intravenous humanised IgG antibody use for prophylaxis of infection in preterm $(<37$ weeks) and low birth weight $(<2500 \mathrm{~g})$ infants were reviewed from 1986 to present. Science Direct, Medline, PubMed and Google Scholar were used to retrieve studies using keywords 'Neonatal sepsis', 'Immunoglobulins', 'Immune-modulation', 'Prophylaxis', 'Neonatal infection', 'Hypogammaglobinaemia', 'Systemic infections', 'Primary Immunodeficiency'. Studies which reviewed IVIG therapy in the treatment of infection, where IgG was administered intramuscularly and where the population was outside the definitions of low birth weight or preterm neonates were all excluded.

Results From our review, we note that the literature reviewed concluded that there was either no reduction to a very marginal reduction in infection rates in neonates.

It is conclusive that while IVIG may potentially confer some benefit in infection prophylaxis and potentially other subtler benefits, it does not demonstrate adequate prophylactic properties to justify routine use in preterm low birth weight neonates.

Conclusion While IVIG use is successful in treating hypogammaglobinaemia (low serum Ig) in primary immune deficiencies, IVIG does not confer the same benefits in infection prophylaxis for neonates. Reasons for this may be attributable to the complexity of the interactions between neonatal immunity and neonatal pathogens. Further research to better understand the mechanisms underlying immune deficiency in preterm and low birth weight infants is advised to offer insight into alternative therapeutic solutions.

\section{GP248 MESENCHYMAL STEM CELL THERAPY IN MICROVILLUS INCLUSION DISEASE}

${ }^{1}$ Ozge Surmeli Onay, 'Ayse Neslihan Tekin*, 'Damla Gunes, 'Ozge Aydemir, ${ }^{2}$ Sevilhan Artan, ${ }^{3}$ Yusuf Aydemir. ${ }^{1}$ Eskisehir Osmangazi University Medical Faculty Department of Pediatrics, Division of Neonatology, Eskisehir, Turkey; ${ }^{2}$ Eskisehir Osmangazi University Medical Faculty Department of Medical Genetics, Eskisehir, Turkey; ${ }^{3}$ Eskisehir Osmangazi University Medical Faculty Department of Pediatrics, Division of Gastroenterology and Hepatology, Eskisehir, Turkey

10.1136/archdischild-2019-epa.307

Objective Microvillus inclusion disease (MVID; MIM $\# 251850)$, is a rare life-threatening secretory and malabsorptive diarrhea of infancy due to mutations in the Myosin 5B (MYO5B) gene. Most of the patients die before achieving the opportunity of bowel transplantation due to electrolytes and renal tubular function disturbances and complications of parenteral nutrition. In this case report, results of stem cell therapy in a newborn infant with MVID were presented and discussed with the relevant literature.

Case report A 6-day-old male patient was referred to our NICU for profuse diarrhea beginning on the postnatal 2nd day after birth, metabolic acidosis and hyponatremia. He was born at $36^{+4}$ gestational weeks as the fourth child of nonconsanguineous parents. Second and third siblings died at 2 and 3,5 months due to complications of intractable diarrhea. Third child's exom sequencing identified a homozygous, disease-causing nonsense variant C.4399C $>T$ (p.Gin1467*) in the MYO5B gene. Both parents were heterozygous for the mutation and they did not accept prenatal diagnosis. Our patient was also affected by homozygous mutation in MYO5B gene. With the knowledge of past history, total parenteral nutrition was started and maximum effort was directed to maintain fluid and electrolyte equilibrium. Patient had high fluid $(350 \mathrm{ml} / \mathrm{kg} / \mathrm{day})$, sodium $(20-25 \mathrm{meq} / \mathrm{kg} / \mathrm{day})$ and bicarbonate requirements $(12-18 \mathrm{meq} / \mathrm{kg} / \mathrm{day})$. On $110^{\text {th }}$ day of life mesenchymal stem cells $\left(1 \times 10^{6}\right.$ Ü transduodenal and $2 \times 10^{6}$ Ü IV) were administered. Although fluid and electrolyte requirements did not decrease after 2 months of follow up after stem cell therapy, the rate of blood stream infections was reduced.

Discussion and Conclusion In MVID, mutations in the MYO5B gene which encodes a protein called myosin $\mathrm{Vb}$ contribute to the dysfunction of enterocytes. Survival rate is less than $25 \%$ at the age of 9 months. Mortality rate is also high after bowel transplantation. Recently, stem cell therapy was presented as a promising alternative strategy for overcoming the current limitations of intestinal failure treatment. We concluded that, the reason why our patient did not benefit from the treatment was rapid turnover of the intestine epithelial cells and the lack of receptive surface caused by the genetic defect. Furthermore, using modified intestinal stem cells instead of mesenchymal stem cells may be more beneficial. In our patient, decreased incidence of infection with probable immunomodulatory effects of stem cells was the secondary gain.

\section{GP249 WHITE CELL COUNTS IN EARLY-ONSET NEONATAL SEPSIS - ARE THEY USEFUL?}

Sean Armstrong*, Ciara Terry, Richard Drew. Rotunda Hospital, Dublin, Ireland

\subsection{6/archdischild-2019-epa.308}

Aims We compared newborn infants with positive blood cultures to a control group of negative cultures in order to establish the usefulness of WCC and Neutrophil values in predicting culture-positive Early-Onset Sepsis.

Methods All positive cultures less than 48hours from birth in infants born in the Rotunda from 2001 to 2017 were identified. WCC and neutrophil values at the time of culture were recorded, and compared to a similar cohort of infants aged less than 48hours with negative blood cultures from 2001-2017. Data was analysed using MedCalc software. 